\title{
Morphology and physiology together: Is optical coherence tomography the one-stop-shop of invasive cardiology?
}

\author{
Carlo Di Mario, Pierluigi Demola \\ Structural Interventional Cardiology, Careggi University Hospital, Florence, Italy
}

Article p. 350

In 1974 Lance Gould demonstrated that maximal coronary flow was progressively reduced by applying external constrictors in animal models [1] and physiology became the gold standard to assess the severity of coronary artery disease. Fractional flow reserve (FFR), measured with invasive intracoronary pressure measurements,

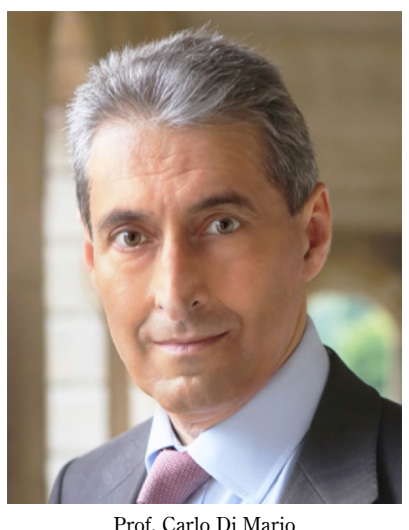

Prof. Carlo Di Mario

difference being the need of urgent revascularization $(11.1 \%$ vs. $1.6 \%$, $\mathrm{p}<0.001)$. A meta-analysis including other trials of FFR-guided treatment of non-culprit lesions confirmed a significant improvement also for the classical "hard" endpoints of MI and death [4].

Gould and Kirkeeide were also pioneers in confirming in vivo, that all multiple parameters contained in the Pouseille law affects stenosis severity, and not only in a minimal area. Intracoronary imaging with intravascular proved to obtain the same goal and became the accepted method to study intermediate coronary lesions (European Society of Cardiology Class I and American College/American Heart Association Class IIa, both with Level of Evidence A). The results of the randomized FFR trials supporting this strong recommendation gained more interest in the past few months based on the unexpected findings of the International Study of Comparative Health Effectiveness with Medical and Invasive Approaches (ISCHEMIA) trial [2]. This large study used non-invasive methods to detect ischemia, especially with single-photon emission computed tomography gave equivocal results with no mortality advantage and a significant, albeit small, reduction in spontaneous myocardial infarction (MI) at follow-up. Using invasive measurements to detect functionally significant stenoses (FFR $\leq 0.80)$ like in The Fractional Flow Reserve versus Angiography for Multivessel Evaluation 2 trial (FAME II trial) patients had an improved outcome with percutaneous coronary intervention (PCI) along with optimal medical therapy [3], with the most striking ultrasound (IVUS) or optical coherence tomography (OCT) overcomes most of the limitations of angiography, with precise luminal measurements which avoid overlapping, foreshortening, and the other limitations of a lumenogram. Unfortunately, the comparison between minimal luminal area (MLA) with OCT and IVUS proved to be poorly correlated with FFR. Conceptually, defining an intermediate stenosis as critical or not in a binary fashion using a dichotomic cut off of MLA is wrong because it does not consider vessel size and lesion length that affect flow dynamics of a lesion. Paradoxically, the first results using a more sophisticated reconstruction based on a morphologic technique to estimate stenosis severity came from quantitative coronary angiography. Conceptually, this correlation can be much better when the lumen measurements are automatically performed with a technique such as OCT that is inherently three-dimensional and where the sharp contour between lumen, cleared by contrast injection, and wall allows fully automated measurements.

Address for correspondence: Pierluigi Demola, MD, Structural Interventional Cardiology, Careggi University Hospital, Florence, Italy, tel: +39348 8421631, e-mail: pierluigidemola@gmail.com 
Optical coherence tomography can detect with accuracy calcium (arc, length and thickness), dissections, stent malposition and helps interventional cardiologists to select the best stent size and length to achieve post-deployment optimization. Is there a need to start OCT only when it is learned from FFR that a lesion needs treatment or can it be used as a one-stop-shop for obtaining all the information needed to decide what to treat and how to do it?

In this issue of 'Cardiology Journal' Gutiérrez-Chico et al. [5] presents a series of 60 patients (76 vessels) studied with FFR because of the presence of intermediate stenoses also receiving an OCT assessment using an algorithm applying flow dynamics to calculate an analogue functional parameter to FFR called optical flow ratio (OFR). OFR showed the same average values of FFR $(0.83 \pm$ \pm 0.09 ) with a clinically relevant disagreement (nonsignificant FFR $\geq 0.80$ with critical $<0.80$ OFR or vice versa) in only $4(5.3 \%)$ cases. It sounds like a striking correlation between a pressure based and an imaging based index of stenosis severity and the authors should be complimented in confirming that lower sampling rates with OCT did not modify this agreement, that simpler OCT based indices such as MLA were poor predictors and that OFR had very low intraand inter-observer variability [6].

The recent comparisons between FFR and the most widely validated non-hyperemic index i.e. iFR and among the many new non-hyperemic indices showed that these correlations are critically dependent on the scattering of data around the cut-point used ( 0.80 for FFR, 0.90 with iFR) [7]. In this study $44 \%$ of FFR measurements were between 0.75 and 0.85 that means the majority of data were not within the brackets of a functionally intermediate lesion. A more stringent comparison with most data close to the cutoff would help in validating the index but the ultimate end-point, as in DEFINE-FLAIR and FFR SWEDEHEART, was a clinical follow-up endpoint $[8,9]$ that requires a much larger sample population. For this study, it was essential to use the non-hyperemic index iFR as a gold standard, modifying the OFR algorithm to perform the estimation in the presence of a baseline flow, and to also repeat the comparison at the end of the procedure. By doing this, an as- sessment of individual importance of single lesions in serial stenosis or diffuse disease can facilitate procedural planning and confirm that a normal or the best possible physiology was achieved at the end of the PCI.

\section{Conflict of interest: None declared}

\section{References}

1. Gould KL, Lipscomb K, Hamilton GW. Physiologic basis for assessing critical coronary stenosis. Instantaneous flow response and regional distribution during coronary hyperemia as measures of coronary flow reserve. Am J Cardiol. 1974; 33(1): 87-94, doi: 10.1016/0002-9149(74)90743-7, indexed in Pubmed: 4808557.

2. Maron DJ, Hochman JS, Reynolds HR, et al. ISCHEMIA Research Group. Initial Invasive or Conservative Strategy for Stable Coronary Disease. N Engl J Med. 2020; 382(15): 1395-1407, doi: 10.1056/NEJMoa1915922, indexed in Pubmed: 32227755.

3. De Bruyne B, Pijls NHJ, Kalesan B, et al. FAME 2 Trial Investigators. Fractional flow reserve-guided PCI versus medical therapy in stable coronary disease. N Engl J Med. 2012; 367(11): 991-1001, doi: 10.1056/NEJMoa1205361, indexed in Pubmed: 22924638.

4. Zimmermann FM, Omerovic E, Fournier S, et al. Fractional flow reserve-guided percutaneous coronary intervention vs. medical therapy for patients with stable coronary lesions: meta-analysis of individual patient data. Eur Heart J. 2019; 40(2): 180-186, doi: 10.1093/eurheartj/ehy812, indexed in Pubmed: 30596995.

5. Gutiérrez-Chico JL, Chen Y, Yu W, et al. Diagnostic accuracy and reproducibility of optical flow ratio for functional evaluation of coronary stenosis in a prospective series. Cardiol J. 2020; 27(4): 350-361, doi: 10.5603/CJ.a2020.0071, indexed in Pubmed: 32436590.

6. Cook CM, Jeremias A, Petraco R, et al. Fractional flow reserve/ instantaneous wave-free ratio discordance in angiographically intermediate coronary stenoses: an analysis using doppler-derived coronary flow measurements. JACC Cardiovasc Interv. 2017; 10(24): 2514-2524, doi: 10.1016/j.jcin.2017.09.021, indexed in Pubmed: 29268881.

7. Jeremias A, Davies JE, Maehara A, et al. Blinded physiological assessment of residual ischemia after successful angiographic percutaneous coronary intervention: the DEFINE PCI study. JACC Cardiovasc Interv. 2019; 12(20): 1991-2001, doi: 10.1016/j. jcin.2019.05.054, indexed in Pubmed: 31648761.

8. Davies JE, Sen S, Dehbi HM, et al. Use of the instantaneous wave-free ratio or fractional flow reserve in PCI. N Engl J Med. 2017; 376(19): 1824-1834, doi: 10.1056/NEJMoa1700445, indexed in Pubmed: 28317458.

9. Escaned J, Ryan N, Mejía-Rentería H, et al. Safety of the deferral of coronary revascularization on the basis of instantaneous wave-free ratio and fractional flow reserve measurements in stable coronary artery disease and acute coronary syndromes. JACC Cardiovasc Interv. 2018; 11(15): 1437-1449, doi: 10.1016/j. jcin.2018.05.029, indexed in Pubmed: 30093050. 